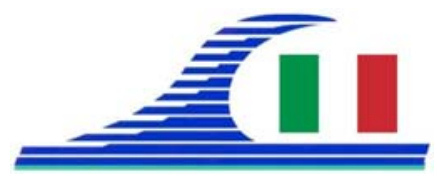

Conférence Méditerranéenne Côtière et Maritime EDITION 3, FERRARA, ITALIA (2015)

Coastal and Maritime Mediterranean Conference

Disponible en ligne - http://www.paralia.fr - Available online

\title{
Etude océanographique de la future centrale électrique, cap Djinet, Wilaya de Boumerdes, Algérie
}

\section{Mohamed Amine ELHADDAD ${ }^{1}$, Adel ZERMOUT ${ }^{1}$, Hamid BOUALI ${ }^{1}$}

1. Laboratoire d’Etudes Maritimes LEM/ingénieur Instrumentation,

Djenane El Malek BP 848 Hydra, Alger, Algérie.

amine.elhaddad@lem-dz.com ; adel.zermout@lem-dz.com ;

hamid.bouali@lem-dz.com

\section{Résumé :}

Durant les cinq dernières années, le site de Cap Djinet a fait l'objet d'une multitude d'études combinées, citons la bathymétrie, l’océanographie, la sismique réflexion et le levé au sonar latéral. L’objectif de ces études était la mise en relief et la compréhension de la configuration géologique du fond marin ainsi que le suivi des paramètres océanographiques dans la zone d'étude.

Le présent document vise à décrire les études océanographiques qui ont été menées en mars 2013, à proximité de la centrale électrique de Cap Djinet. Plusieurs levés ont été réalisés sur ce site où différents instruments ont été déployés et cela pour la collecte des données océanographiques à savoir : la marée, la vitesse et la direction des courants, la température, la salinité de l'eau de mer. Les données ainsi collectées ont servi à l'établissement d'un modèle numérique dont l'objectif est d'implanter une nouvelle prise d'eau et de l'extension de la centrale électrique de Cap Djinet.

Mots-clés : Bathymétrie, Océanographie, Sismique réflexion, Sonar latéral, Géologie, Marée, Courant, Température, Salinité, Modèle numérique.

\section{Introduction}

En mars 2013, le laboratoire d'études maritime a été sollicité pour mener une large campagne océanographique au voisinage de la centrale électrique de Ras Djinet, une région qui se trouve à environ $60 \mathrm{~km}$ à l'est de la capitale Alger. La campagne s'inscrit dans le cadre de l'étude pour la réalisation d'une autre prise d'eau et l'extension de la centrale électrique existante.

Les travaux de cette campagne sont consignés dans la présente publication qui décrit la consistance des travaux, les moyens mis en place, la méthode utilisée ainsi que les données océanographiques collectées, ces dernières ont été exploitées et ont servies de paramètres d'entrer pour la modélisation; conception d'un modèle numérique de la zone d'étude à proximité de la centrale électrique. 
Côtes méditerranéennes menacées :

Risques et défis dans le contexte du changement climatique

\section{Matériels et méthodes}

\subsection{Matériels}

La campagne océanographique a nécessité la participation et la coordination d'un personnel scientifique pluridisciplinaires, à savoir: des ingénieurs électroniciens spécialisés dans l'instrumentation côtière, océanographes, plongeurs, ...

Les équipements et les enregistreurs ayant été utilisés dans le cadre de cette campagne sont :

- Un système de positionnement, GPS hémisphère.

- Deux Courantomètres ADCP Workhorse fabriqués par RD Instruments.

- Quatre enregistreurs de salinité et de température SeaBird SBR37 MicroCat CTD.

- Deux marégraphes type VALPORT 740.

- Deux enregistreurs de pression type RBR TGR-1050 et RBR DR-1050.

- Logiciels d'acquisition et de traitement de données.

- Matériel de prélèvements et de conservation d'échantillons d'eau et de sédiment.

\subsection{Méthodes}

En phase préalable, l'ensemble des données bathymétriques disponibles couvrant la zone d'étude, a été exploité afin de pouvoir déterminer avec précision les profondeurs auxquelles les enregistreurs seront déployés; enregistreurs CTD, ADCPS, etc. (LABORATOIRE D’ETUDES MARITIMES, 2010).

Après avoir déterminé les paramètres océanographiques à enregistrer, une étude a été faite visant la préparation et la mise en place les systèmes de fixation et les lignes de mouillage constituant les enregistreurs, en tenant compte des différentes forces et contraintes auxquelles les enregistreurs seront soumis durant toute la durée de déploiement de ces derniers (GIRARDOT, 2001).

Il a été procédé par la suite à la configuration de tous les équipements (cadence d'enregistrement, durée d'acquisition, ...).

A l'issue des opérations suscitées, tous les équipements ont été déployés en mer pour une durée d'acquisition d'un mois, les principales activités de la campagne sont :

i. Mesures en continue de la marée.

ii. Mesures en continu du courant et de la houle.

iii. Mesures en continu de la température et de la salinité de l'eau de mer.

D’autres campagnes ont été menées en parallèle à savoir :

iv. Trois campagnes de mesure en temps réel de la distribution horizontale du courant.

v. Deux campagnes de mesure en temps réel de profiles de température et de salinité.

vi. Prélèvement d'échantillons d'eau et de sédiment pour analyse en laboratoire.

La figure 1 illustre les positions des mesures en continu et en temps réel des données océanographique dans la zone d’études. 


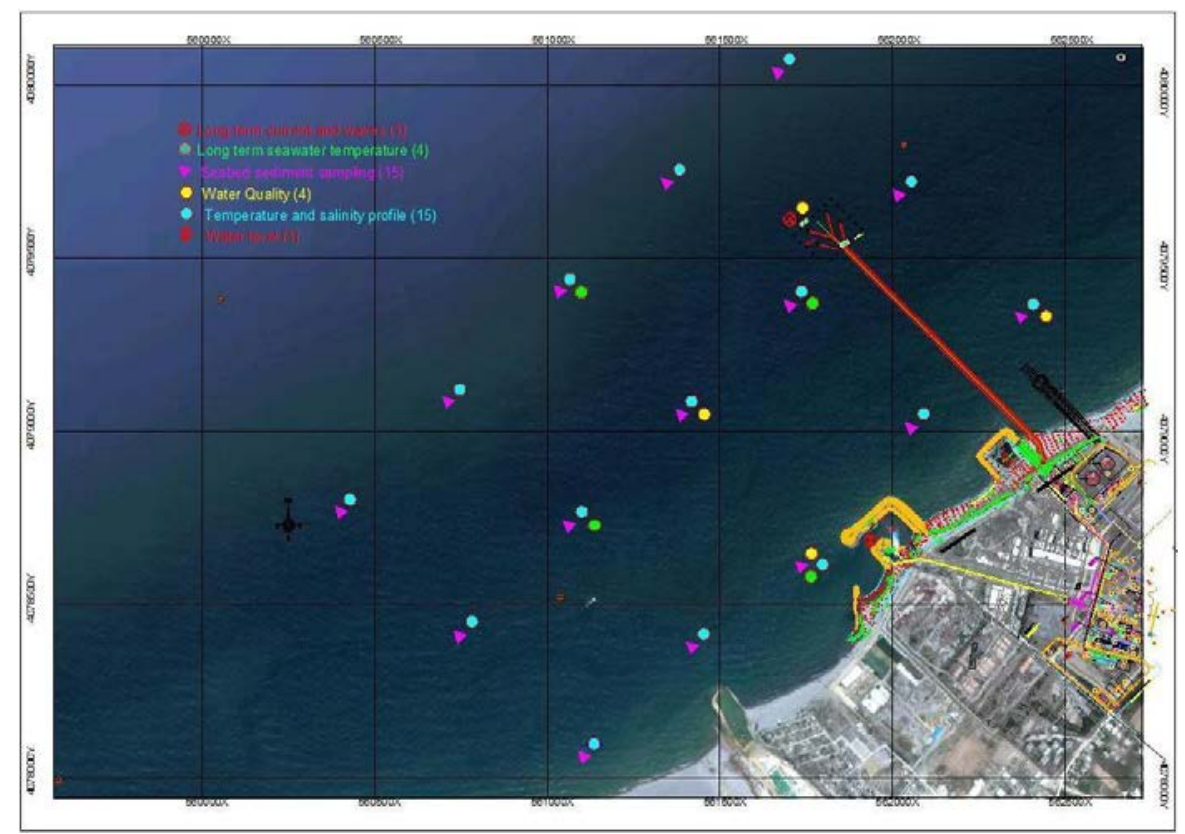

Figure 1. La zone d'étude montrant les positions pour la mesure en continu et en temps réel des données.

\section{Résultats et interprétation}

A l'issue de la campagne océanographique, tous les équipements déployés sur site ont été récupérés, les données brutes ont été traitées en utilisant pour cela des logiciels appropriés.

Des analyses harmoniques et statistiques de la marée ont montré les faibles amplitudes du niveau d'eau au voisinage de la zone d'étude. Cela a engendré de très faibles valeurs du courant marin durant les trois campagnes de mesure. La figure 2 illustre le niveau de la marée au voisinage du site d'étude.

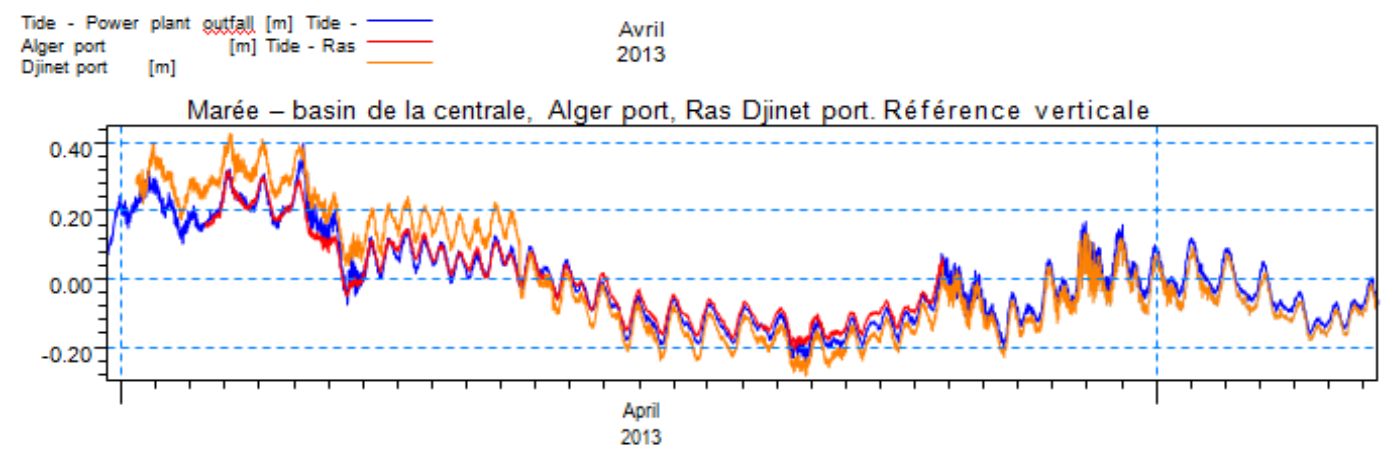

Figure 2. Niveau de la marée au voisinage du site d'étude.

Le traitement des données a permis la compréhension du comportement du courant marin et de la houle (vitesse et direction) ainsi que la détermination de la stratification thermique tout a long de la zone d'étude. 
Côtes méditerranéennes menacées :

Risques et défis dans le contexte du changement climatique

La figure 3 illustre les résultats de la deuxième campagne de mesure en temps réel de la distribution du courant horizontale au voisinage de la zone d'étude.

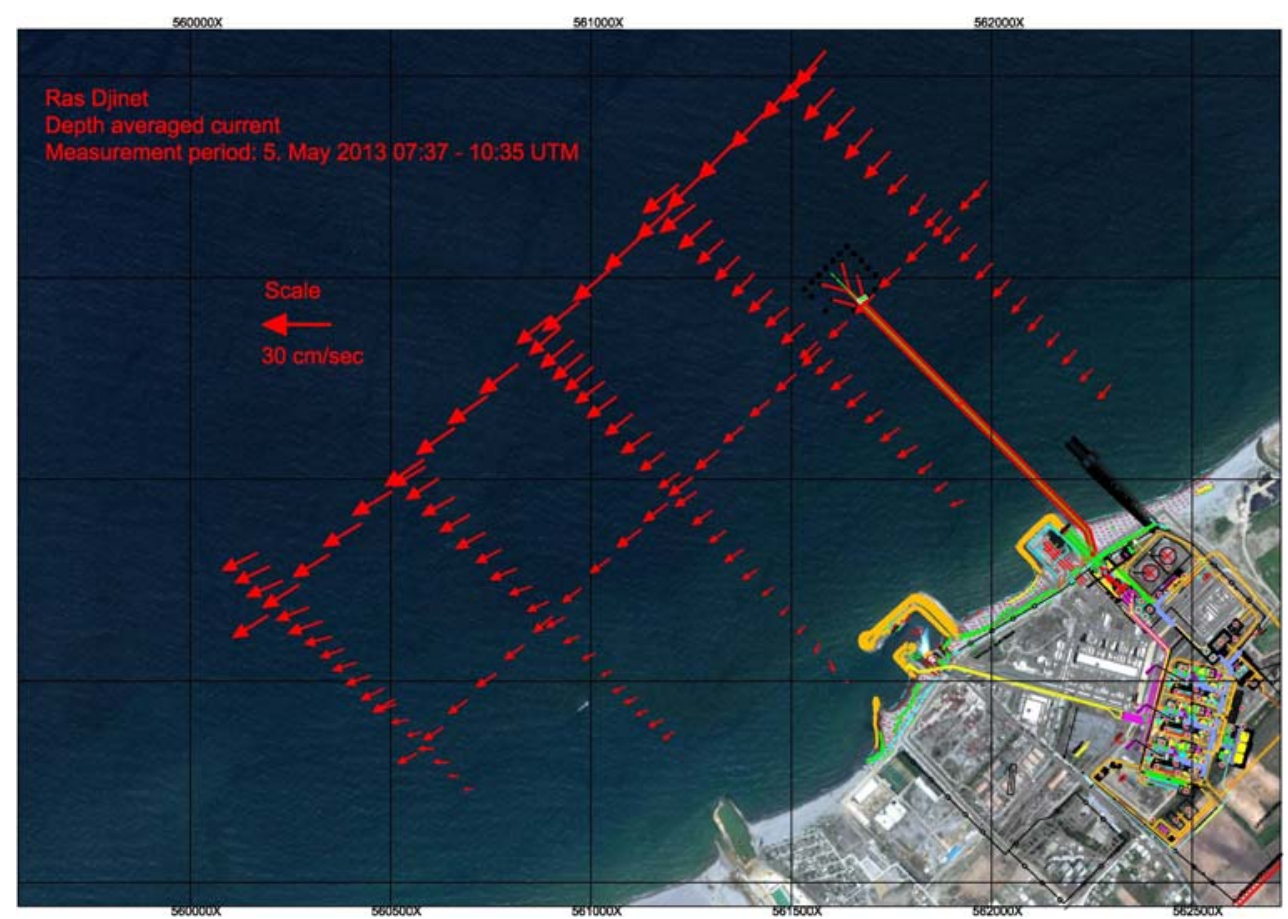

Figure 3. Un exemple de présentation du courant horizontal de la deuxième compagne de mesure.

\section{Conclusions}

Les résultats de l'étude sont fortement tributaires de la qualité des données hydrographiques et de la collecte en temps opportun de ces données.

Les résultats de la campagne océanographique menée à Cap Djinet, ont permis d'une part, de cerner tout un ensemble de données hydrographiques (direction et vitesse du courant et de la houle, la marée, la température de l'eau, les prélèvements des échantillons d'eau et de sédiment, ...) jugées nécessaires pour la calibration et le test du modèle numérique, ainsi que l'évaluation de l'invasion et de la dispersion du sédiment à travers la zone d'étude d'autre part. Cette évaluation est fondamentale pour la mise en place des systèmes de la prise d'eau et du rejet ainsi que leurs structures appropriées.

\section{Références bibliographiques}

GIRARDOT J.-P. (2001). Techniques des mouillages utilisés en océanographie. Rapport du Laboratoire de Physique des Océans, Université de Bretagne Occidentale, 55 p.

LABORATOIRE D'ETUDES MARITIMES (2010). Études océanographique de la future centrale électrique de Cap Djinet. Rapport \& plan du levé topo-bathymétrique. 